\title{
Late-onset distant metastases confer poor prognosis in patients with well-differentiated thyroid cancer
}

\author{
Chan Kwon Jung ${ }^{1,2}$, Sohee Lee ${ }^{3}$, Ja Seong Bae ${ }^{3}$, Dong-Jun Lim ${ }^{4}$ \\ ${ }^{1}$ Department of Hospital Pathology, ${ }^{2}$ Cancer Research Institute, ${ }^{3}$ Department of Surgery, ${ }^{4}$ Division of Endocrinology and Metabolism, Department \\ of Internal Medicine, College of Medicine, The Catholic University of Korea, Seoul, Korea \\ Contributions: (I) Conception and design: All authors; (II) Administrative support: CK Jung; (III) Provision of study materials or patients: All authors; \\ (IV) Collection and assembly of data: All authors; (V) Data analysis and interpretation: All authors; (VI) Manuscript writing: All authors; (VII) Final \\ approval of manuscript: All authors. \\ Correspondence to: Chan Kwon Jung, MD, PhD. Department of Hospital Pathology, Seoul St. Mary's Hospital, College of Medicine, The Catholic \\ University of Korea, 222 Banpo-daero, Seocho-gu, Seoul 06591, Korea. Email: ckjung@catholic.ac.kr.
}

Background: Distant metastases from well-differentiated thyroid carcinoma (WDTC) occasionally occur over a wide range of time intervals after primary thyroid surgery. The prognostic impact of the timing of distant metastasis onset remains unclear.

Methods: We retrospectively reviewed the clinicopathologic features and clinical outcomes of 57 patients with WDTC and distant metastases, and evaluated the mutational profiles of BRAF, RAS, and TERT promoter genes. All patients underwent thyroidectomy and radioactive iodine (RAI) ablation using the same treatment protocol. Synchronous distant metastases were defined as those detected within 12 months of the primary WDTC diagnosis. Metachronous metastases were considered early- and late-onset diseases if detected $1-5$ and $\geq 5$ years after the primary diagnosis, respectively.

Results: In all patients, the 5- and 10-year cancer-specific survival (CSS) rates after the diagnosis of distant metastasis were $86 \%$ and $57 \%$, respectively. Late-onset ( $\geq 5$ years) metachronous distant metastasis was associated with age of $\geq 55$ years $(\mathrm{P}=0.043)$ and patients refractory to RAI therapy $(\mathrm{P}=0.026)$. TERT promoter mutations were associated with RAI refractivity $(\mathrm{P}=0.026)$. BRAF V600E and RAS mutations had no prognostic significance. Bone metastasis $(\mathrm{P}=0.002)$ and the onset time of distant metastasis $(\mathrm{P}=0.004)$ were associated with poor CSS. There was no significant difference in CSS between patients with synchronous distant metastases and those with early-onset (1-5 years) metachronous distant metastases. In the multivariate analysis, bone metastasis [hazard ratio $(\mathrm{HR})=10.24 ; 95 \%$ confidence interval (CI): 1.25-83.74; $\mathrm{P}=0.030$ ] and late-onset ( $\geq 5$ years) metachronous distant metastasis (HR =5.20; 95\% CI: 1.01-26.63; $\mathrm{P}=0.048$ ) were independent predictors for worse CSS.

Conclusions: The prognosis of patients with WDTC was poorer for late metachronously detected metastases than for synchronous or early metachronous metastases. Patients with distant metastasis occurring 5 years later after primary thyroid diagnosis should, therefore, be more carefully treated.

Keywords: Thyroid cancer; papillary carcinoma; follicular carcinoma; distant metastasis; synchronous; metachronous; prognosis

Submitted Mar 27, 2020. Accepted for publication Jun 19, 2020.

doi: $10.21037 /$ gs-20-416

View this article at: http://dx.doi.org/10.21037/gs-20-416

$\wedge$ ORCID: 0000-0001-6843-3708. 


\section{Introduction}

Well-differentiated thyroid carcinoma (WDTC), comprising the majority of thyroid cancers, includes papillary thyroid carcinoma (PTC) and follicular thyroid carcinoma (FTC). Although thyroid cancer is generally an indolent disease that has a much lower mortality rate than other solid organ cancers (1), about 2,000 deaths from thyroid cancer occur in the USA annually (The American Cancer Society's estimates for thyroid cancer in the United States for 2020, www.cancer.org). Distant metastasis is the leading cause of cancer-related death and occurs in $1-3 \%$ of the WDTC patients at the initial diagnosis and in 6-23\% of the cases during the follow-up period after surgery (1-4). The pathologic parameters associated with the distant metastasis are aggressive histologic variants, large tumor size, lateral lymph node metastasis, and extensive vascular invasion $(1,5,6)$. The presence of TERT promoter mutations is strongly associated with distant metastasis in patients with WDTC (7-10).

WDTC may develop late-onset distant metastasis after primary treatment, with some cases emerging several decades later (5). The metachronous distant metastasis of WDTC is usually defined as an occurrence of the disease at distant organs later than 12 months after the primary diagnosis $(3,11)$. The prognostic significance of the timing of the appearance of distant metastases remains controversial. A previous study demonstrated that WDTC patients with metachronous distant metastasis had a shorter cancer-related survival time than those with synchronous distant metastasis (3). However, the prognostic differences according to the times to metastasis for distant organs were not significant in other studies (11-13). While distant metastases can occur decades after initial treatment, little is known about the prognostic implication of a metachronous distant metastasis occurring after a long latent interval following the initial treatment of WDTC.

This study aimed to investigate the prognostic significance of late-onset of distant metastasis and identify the clinicopathological and molecular features for predicting the prognosis of WDTC patients with distant metastasis. We present the following article in accordance with the STROBE reporting checklist (available at http://dx. doi. org/10. 21037/gs-20-416).

\section{Methods}

This retrospective study was approved by the institutional review board of Seoul St. Mary's Hospital of the Catholic University of Korea (KC16SISI0709) and conducted in accordance with the Declaration of Helsinki. Informed consent was obtained from each patient included in the study.

We retrospectively reviewed 57 WDTC patients with distant metastases who were treated at Seoul St. Mary's Hospital between 2006 and 2019. The same data set was used in our recent study (14), but clinical follow-up data were updated as of March 2020.

All distant metastases were confirmed by pathologic examination when available and imaging modalities, including computed tomography, magnetic resonance imaging, whole-body scans, and positron-emission tomography. All patients underwent initial total thyroidectomy or two-stage thyroidectomy (initial lobectomy and subsequent completion thyroidectomy), and then radioactive iodine (RAI) therapy once or several times depending on the course of the disease. The pathologic diagnosis of WDTC was as follows: 48 PTCs (26 classic, 11 tall cell, five follicular, two columnar cell, two diffuse sclerosing, one solid, and one hobnail variant) and nine FTCs (eight encapsulated angioinvasive and one minimally invasive type). We excluded cases showing morphologic evidence for tumoral transformation to poorly differentiated and anaplastic carcinoma (e.g., high mitotic activity, necrosis, solid/trabecular/insular growth pattern, or cellular pleomorphism) in the primary or metastatic tumors.

Refractoriness to RAI therapy was defined as at least one of the following four criteria (15): (I) the presence of at least one distant lesion with no detectable RAI uptake, (II) progressive disease despite previous RAI treatment and confirmed uptake, (III) progressive disease within 1-year after RAI therapy, and (IV) persistent disease after the administration of a cumulative dose of RAI $\geq 600 \mathrm{mCi}$.

\section{Thyroglobulin (Tg) doubling-time}

Tg doubling time was calculated using serum $\mathrm{Tg}$ levels measured under thyrotropin suppression (TSH $<0.1 \mathrm{mIU} / \mathrm{L}$ ) according to the $\log$-slope method as previously described (16). Twelve patients were excluded due to presence of anti-Tg antibody $(n=8)$ or less than four data of serum $\mathrm{Tg}$ measurement under TSH suppression $(\mathrm{n}=4)$. Zero time point was defined as the first $\mathrm{Tg}$ measurement day after the detection of distant metastasis. We used a Microsoft Excel-based doubling time calculator offered by Kuma Hospital (www.kuma-h.or.jp). 


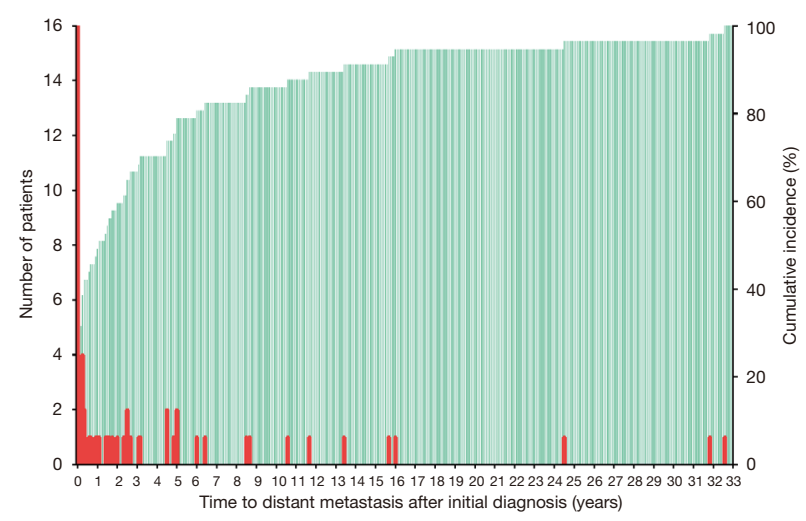

Figure 1 Frequency distribution (bars, left Y-axis) and cumulative proportions (area, right Y-axis) of patients with WDTC according to the onset time of distant metastasis. WDTC, well-differentiated thyroid carcinoma.

\section{Molecular analysis}

Genomic DNA was isolated from the paraffin-embedded tumor tissue blocks of 36 primary thyroid tumor tissues and 21 metastatic lesions. Sanger sequencing for the $B R A F$ (codon 600), three types of RAS [NRAS, HRAS, and KRAS (codons 12, 13 and 61)] genes, and TERT promoter (124 and 146 bp upstream of the translation start site) was performed as previously described (17-20).

\section{Statistical analysis}

The patients with distant metastasis were classified into three groups according to the timing of its appearance as synchronous (within 12 months from the primary diagnosis of WDTC), early-onset metachronous (between 1 and 5 years after the initial diagnosis), and late-onset metachronous ( 5 years or later after the initial thyroid diagnosis).

The statistical significance of the categorical variables was tested with a Chi-squared or Fisher's exact test. The cancer-specific survival (CSS) time was defined the survival time in the absence of cancer-related death and calculated from the onset of distant metastasis. The Kaplan-Meier method was used to plot the CSS curves. Statistical differences between the curves were tested by the logrank test. Cox proportional-hazards model was used to analyze the multivariate-adjusted hazard ratios (HRs) and $95 \%$ confidence intervals (CIs) of the predictive variables in terms of CSS. Statistical analyses were performed using SPSS (version 21.0, IBM Corp, Armonk, NY, USA).
Survival curves were generated using GraphPad Prism (version 6.05, GraphPad Software, La Jolla, CA, USA). $\mathrm{P}$ values of less than 0.05 were considered statistically significant.

\section{Results}

The median age at the detection of distant metastasis was 59 (range, 14-77) years. Lung metastases (95\%) and bone metastases (33\%) occurred most frequently. Of all 57 WDTC patients, 28 (49\%) developed distant metastasis as the initial manifestation or within 12 months after the primary diagnosis. In 29 patients with metachronous distant metastasis, the median time from the initial diagnosis to the detection of distant metastasis was 32 months and the maximum interval to the appearance of distant metastasis was 32 years (Figure 1). The clinicopathologic characteristics of the 57 WDTC patients and the onset time of distant metastasis are summarized in Table 1 . The patients with late-onset metachronous distant metastases (occurring $\geq 5$ years after initial diagnosis) were enriched in the older age $(\mathrm{P}=0.043)$ and RAI-refractory groups $(\mathrm{P}=0.026)$.

The median follow-up of all patients after the diagnosis of distant metastases was 48 (range, 3-143) months. Of 57 patients, 21 (40\%) were refractory to RAI and nine (16\%) died of thyroid cancers during the follow-up, while no deaths occurred in RAI-responsive patients. The 5- and 10 -year CSS rates after the diagnosis of distant metastasis were $86 \%$ and $57 \%$, respectively.

$\mathrm{Tg}$ doubling times were classified into three groups according to the period: (I) negative value, (II) $0-3$ years, and (III) $\geq 3$ years, as shown in Table 1 and Figure 2. There was a significantly decreasing trend in rates of patients with negative values of $\mathrm{Tg}$ doubling-time as increasing in the time of appearance of distant metastasis $(\mathrm{P}=0.008)$ (Table 1, Figure 2). De novo anti-Tg antibodies occurred in eight patients who were initially negative for the anti- $\mathrm{Tg}$ antibodies. Cancer-specific death rate was higher in patients with anti-Tg antibodies $(50 \%, 4 / 8)$ than those without the antibodies $(10 \%, 5 / 49)(\mathrm{P}=0.016$, Figure 2$)$.

On univariate analysis, bone metastasis $(\mathrm{P}=0.002)$, RAI refractivity $(\mathrm{P}=0.001)$, and the onset time of distant metastasis $(\mathrm{P}=0.004$, Figure 3$)$ were significantly associated with worse CSS (Table 2). There was no difference in the CSS rates between the patients with synchronous and earlyonset (1-5 years) metachronous distant metastases. An age of $\geq 55$ years at the diagnosis of distant metastasis also showed worse CSS but it was not statistically significant 
Table 1 Clinicopathologic and molecular features of WDTC patients according to the timing of appearance of distant metastases

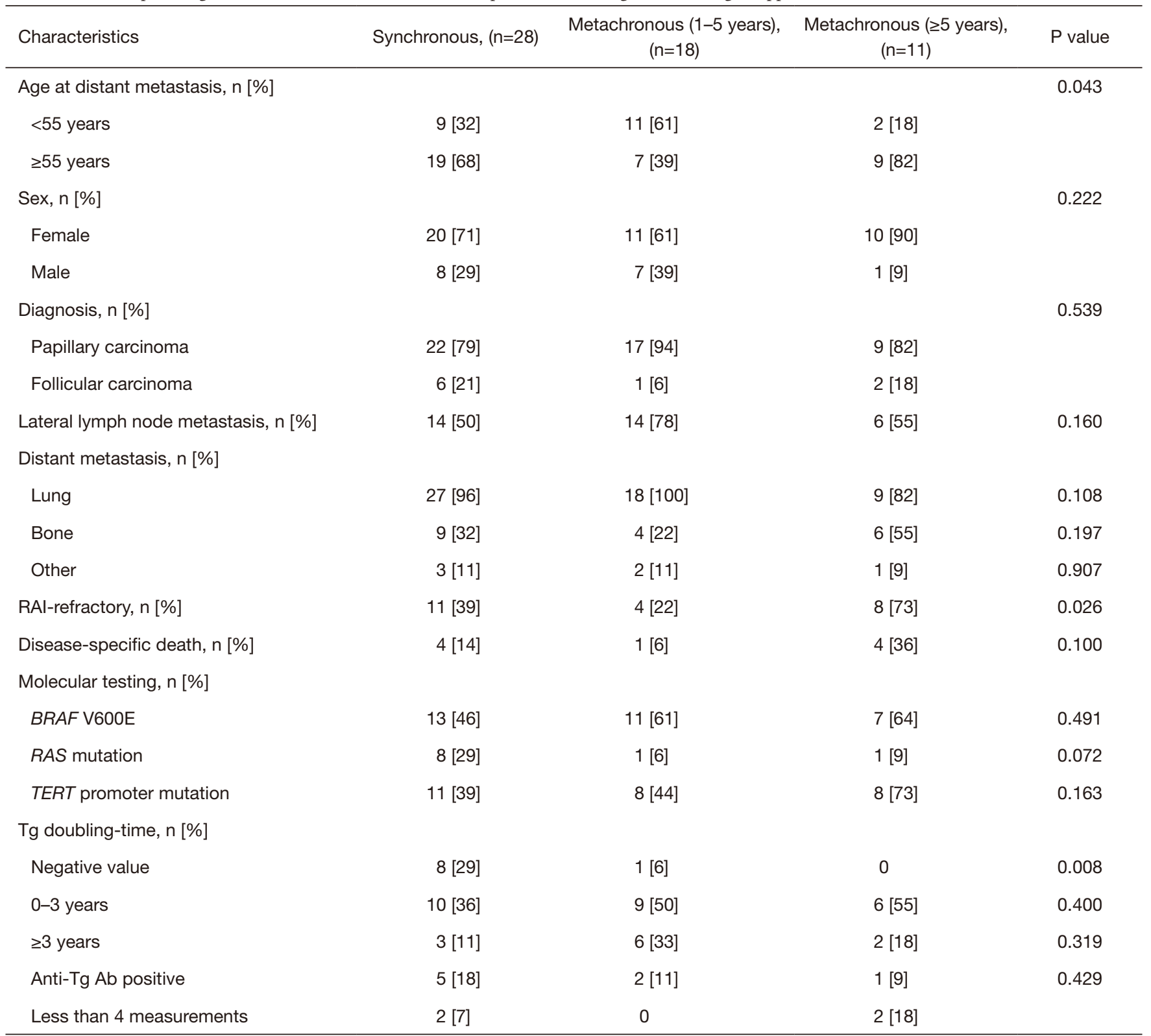

WDTC, well-differentiated thyroid carcinoma; RAI, radioactive iodine; Tg, thyroglobulin; Ab, antibody.

( $\mathrm{P}=0.055)$. The presence of TERT promoter mutations was significantly associated with RAI refractivity $(\mathrm{P}=0.026)$ but was not related to CSS $(\mathrm{P}=0.435)$. The BRAF V600E and $R A S$ mutations had no prognostic significance in WDTC patients with distant metastases. The presence of concurrent mutations in the TERT promoter and BRAF or RAS genes was not associated with CSS $(\mathrm{P}=0.474)$.

In the multivariate analysis, bone metastasis $(\mathrm{HR}=10.24$; 95\% CI: $1.25-83.74 ; \mathrm{P}=0.030)$ and late-onset ( $\geq 5$ years $)$ metachronous distant metastasis (HR $=5.20 ; 95 \%$ CI: $1.01-26.63 ; \mathrm{P}=0.048)$ remained as independent negative predictors for CSS (Table 2).

We further analyzed the mutational profiles of matched primary and metastatic tumor samples in six patients. The $B R A F \mathrm{~V} 600 \mathrm{E}$ and $R A S$ mutations were found in three and two primary thyroid tumors, respectively, and retained in the matched metastasis. In two cases, the TERT promoter mutations were not found in primary thyroid tumors, but 


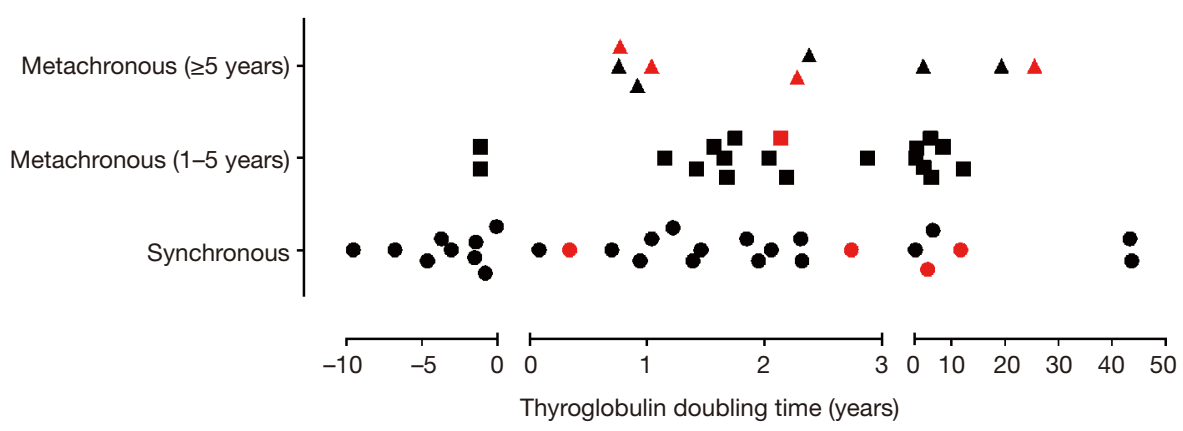

Figure 2 Distribution of values of Tg doubling time in WDTC patients with distant metastasis. Upper, flow chart showing selection of patients for the measurement of $\mathrm{Tg}$ doubling time. Lower, a scatter plot showing relationship between $\mathrm{Tg}$ doubling time and onset time of distant metastasis. Red dots indicate deaths during follow-up. Tg, thyroglobulin; WDTC, well-differentiated thyroid carcinoma.

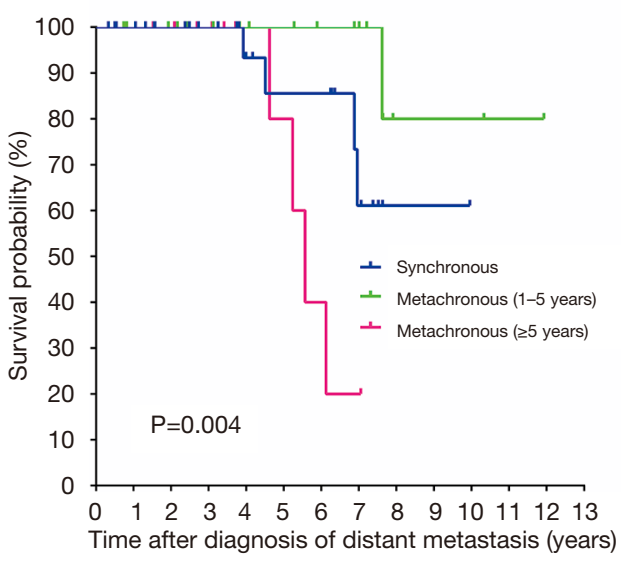

Figure 3 CSS curve of patients with WDTC and distant metastasis according to the onset time of the distant metastasis. Survival times were calculated from the diagnosis of first distant metastasis. CSS, cancer-specific survival; WDTC, well-differentiated thyroid carcinoma.

were present in their matched metastases. In three cases, the TERT promoter mutations were identified in both primary and matched metastatic tumors.

\section{Discussion}

The mortality of WDTC patients is strongly related to old age and distant metastasis. Previous studies have shown that the survival of patients with thyroid cancer may be associated with the onset time of distant metastasis $(3,4,11,21)$. However, the results are controversial, and there have been no studies focusing on further categorizing patients with metachronous distant metastases based on the onset time of distant metastases. In the current study, we showed that patients developing late-onset ( $\geq 5$ years) distant metastasis after a primary thyroid diagnosis had shorter survival times than those developing distant metastasis initially or within 5 years after primary thyroid surgery.

WDTC can dedifferentiate to poorly differentiated and anaplastic thyroid cancer via the acquisition of additional mutations and epigenetic alterations (22-24). WDTC can also behave aggressively without any components of highgrade or dedifferentiated histologic features (22). Patient age is an important prognostic factor for CSS in WDTC (25). Mutation frequency and density in PTC increases with the age of the patients (26). TERT promoter mutations are more frequently found in WDTC patients of older age and associated with distant metastasis, advanced cancer stage, and cancer-specific mortality $(24,27)$. In our study, patients with late-onset ( $\geq 5$ years) metachronous distant metastases were older and had a higher rate of TERT promoter mutations ( $73 \%$ vs. $41 \%$ ) at the diagnosis of metastatic cancer than those with synchronous or earlyonset metachronous distant metastases. These findings may be associated with worse clinical outcomes of patients with late-onset ( $\geq 5$ years) metachronous distant metastasis.

The independent prognostic factors for survival in patients with WDTC include age at diagnosis, tumornode-metastasis (TNM) staging, and TERT promoter mutations (9,28-30). Previous studies have shown that concurrent TERT promoter and $B R A F$ or $R A S$ mutations had synergistic effects on the worse clinical outcomes of the patients with WDTC $(8,9,27,29,31)$. In contrast with previous studies, we only enrolled patients who developed distant metastasis. TERT promoter mutations were predictive of RAI refractivity but was not related to CSS in 
Table 2 Prognostic factors for CSS in WDTC patients with distant metastasis

\begin{tabular}{|c|c|c|c|c|c|}
\hline Characteristics & \multicolumn{3}{|c|}{ Univariate analysis } & \multicolumn{2}{|c|}{ Multivariate analysis } \\
\hline Age at distant metastasis & & & 0.055 & & \\
\hline$<55$ years $(n=22)$ & 92 & 92 & & Reference & \\
\hline$\geq 55$ years $(n=35)$ & 88 & 36 & & $1.54(0.15-15.50)$ & 0.714 \\
\hline Female $(n=41)$ & 100 & 68 & & & \\
\hline Male $(n=16)$ & 70 & 35 & & & \\
\hline Diagnosis & & & 0.094 & & \\
\hline Papillary carcinoma $(n=48)$ & 92 & 62 & & Reference & \\
\hline Absent $(n=23)$ & 89 & 44 & & & \\
\hline Present $(n=34)$ & 90 & 68 & & & \\
\hline Bone metastasis & & & 0.002 & & \\
\hline Absent $(n=37)$ & 100 & 90 & & Reference & \\
\hline Present $(n=19)$ & 75 & 28 & & $10.24(1.25-83.74)$ & 0.030 \\
\hline RAI & & & 0.001 & $\mathrm{NA}^{\dagger}$ & \\
\hline Responsive $(n=34)$ & 100 & 100 & & & \\
\hline Refractory ( $n=23$ ) & 80 & 28 & & & \\
\hline $0-3$ years $(n=25)$ & 80 & 49 & & & \\
\hline Other $(n=20)$ & 100 & 100 & & & \\
\hline De novo anti-Tg antibody & & & 0.088 & & \\
\hline Absent $(n=49)$ & 91 & 75 & & & \\
\hline Present $(n=8)$ & 83 & 22 & & & \\
\hline BRAF V600E & & & 0.827 & & \\
\hline Absent $(n=26)$ & 92 & 65 & & & \\
\hline Present $(n=31)$ & 87 & 48 & & & \\
\hline RAS mutation & & & 0.208 & & \\
\hline Absent $(n=47)$ & 87 & 64 & & & \\
\hline Present $(n=10)$ & 100 & 40 & & & \\
\hline
\end{tabular}

Table 2 (continued) 
Table 2 (continued)

\begin{tabular}{|c|c|c|c|c|c|}
\hline Characteristics & \multicolumn{3}{|c|}{ Univariate analysis } & \multicolumn{2}{|c|}{ Multivariate analysis } \\
\hline TERT promoter mutation & & & 0.435 & & \\
\hline Absent $(n=30)$ & 92 & 73 & & & \\
\hline Present $(n=27)$ & 87 & 51 & & & \\
\hline TERT promoter + (BRAF or RAS) $(\mathrm{n}=22)$ & 83 & 49 & & & \\
\hline Other $(n=35)$ & 94 & 72 & & & \\
\hline
\end{tabular}

patients with distant metastasis. Therefore, further studies are needed to evaluate whether TERT promoter mutations are predictive of cancer mortality in patients with WDTC and distant metastasis.

Most patients with thyroid cancer present with localized (confined to the thyroid) or regional (spread to regional lymph nodes) disease. According to the Surveillance, Epidemiology, and End Results (SEER) Cancer Statistics Review from 1975 through 2017 in the USA, the 5-year conditional relative survival rates for localized and regional thyroid cancer at the time of diagnosis are $99.9 \%$ and $97.8 \%$, respectively, but fall to $54.8 \%$ when distant metastasis is present at diagnosis (32). In our study, the 5- and 10-year CSS rates for WDTC with synchronous distant metastasis were $86 \%$ and $61 \%$. The results are consistent with a recent multicenter study performed in Korea, wherein the 5- and 10-year CSS rates for patients with WDTC and initial distant metastasis were $85 \%$ and $68 \%$ (33). The difference in the survival rates between the Korean and American populations may be explained by differences in the study population (e.g., age, race, ethnicity, or pathology), cancer detection times (early diagnosis of WDTC in Korea), and therapeutic modalities including RAI and multitargeted kinase inhibitors.

Serum Tg doubling time was initially introduced to predict the cancer progression and prognosis of patients with differentiated thyroid cancers (DTC) $(16,34)$. However, a recent study showed that the $\mathrm{Tg}$ doubling time of DTC patients with distant metastasis was not statistically associated with cancer progression (35). In our study, negative values of $\mathrm{Tg}$ doubling time were found predominantly in patients with synchronous distant metastasis. However, positive values of Tg doubling time were not significantly different among the three groups according to the onset time of distant metastasis. As anti$\mathrm{Tg}$ antibodies interfere with the serum $\mathrm{Tg}$ measurement by immunoassay, Tg doubling time should not be calculated in patients with anti-Tg antibodies. In the present study, we found the de novo development of anti-Tg antibodies in $14 \%$ of patients. De novo anti-Tg antibody positivity was marginally associated with CSS (Table 2). In a recent study using a large North American DTC registry, de novo anti-Tg antibody detection after initial treatment was not associated with structural recurrence (36). However, other previous studies reported the association of anti-Tg antibody positivity with the cancer progression or prognosis of DTC patients after initial treatment $(35,37)$. Therefore, further studies are required to determine the clinical significance of $\mathrm{Tg}$ doubling time and anti-Tg antibody positivity in the follow up of DTC patients with distant metastasis.

Bone is the second-most common site of distant metastasis from thyroid cancer after the lungs (2$4,11,21,38,39)$. Bone metastases were associated with worse prognosis in thyroid cancer than lung metastases in many previous studies $(2-4,11,21,38,39)$. Our results also showed that bone metastasis was an independent prognostic factor for the CSS of patients with WDTC.

Our study had several inevitable limitations including the retrospective study design. First, the number of study subjects was too small to permit further analysis in subgroups. Further large-scale studies should be done to validate our results. Second, variable follow-up periods in our study might have caused imprecision and bias because subjects lost to follow-up are inevitable in most cohort 
studies. To overcome the inherent limitation, we doublechecked our mortality data by comparing the data obtained from the Korea Central Cancer Registry and the Korean Statistical Information Service. Third, not all primary thyroid tissues were available for molecular testing because the old tissue blocks had poor quality DNA or were not stored in the pathology files. These limitations may have contributed to a bias that could have affected the study outcomes.

\section{Conclusions}

Despite the relatively indolent nature of WDTC, we observed a 10 -year CSS rate of $57 \%$ in patients who developed distant metastases. The clinical outcomes of patients with WDTC may be affected by the onset time of distant metastasis. Distant metastasis occurring 5 years later after a primary thyroid diagnosis was an independent predictor for poor CSS. The onset time of distant metastasis could be considered as an indicator of the risk stratification and prognosis of patients with WDTC. Further studies should be performed to identify the predictors for a certain subset of WDTC patients who will develop distant metastases later in the course of the disease.

\section{Acknowledgments}

Funding: This research was supported by grants (NRF2017R1D1A1B03029597 and NRF-2020R1F1A1070028) from the Basic Science Research Program through the National Research Foundation of Korea funded by the Ministry of Science and ICT.

\section{Footnote}

Provenance and Peer Review: This article was commissioned by the Guest Editor (Kennichi Kakudo) for the series "Asian and Western Practice in Thyroid Pathology: Similarities and Differences" published in Gland Surgery. The article was sent for external peer review organized by the Guest Editor and the editorial office.

Reporting Checklist: The authors have completed the STROBE reporting checklist. Available at http://dx. doi. org/10. 21037/gs-20-416

Peer Review File: Available at http://dx. doi. org/10. 21037/ gs-20-416
Conflicts of Interest: All authors have completed the ICMJE uniform disclosure form (available at http://dx. doi. org/10. 21037/gs-20-416). The series "Asian and Western Practice in Thyroid Pathology: Similarities and Differences" was commissioned by the editorial office without any funding or sponsorship. CKJ serves as an unpaid editorial board member of Gland Surgery from Jul 2019 to Jun 2021. The authors have no other conflicts of interest to declare.

Ethical Statement: The authors are accountable for all aspects of the work in ensuring that questions related to the accuracy or integrity of any part of the work are appropriately investigated and resolved. The Institutional Review Board of Seoul St. Mary's Hospital of the Catholic University of Korea approved this study (KC16SISI0709). Informed consent was obtained from each patient included in the study. This study was conducted in accordance with the Declaration of Helsinki.

Open Access Statement: This is an Open Access article distributed in accordance with the Creative Commons Attribution-NonCommercial-NoDerivs 4.0 International License (CC BY-NC-ND 4.0), which permits the noncommercial replication and distribution of the article with the strict proviso that no changes or edits are made and the original work is properly cited (including links to both the formal publication through the relevant DOI and the license). See: https://creativecommons.org/licenses/by-nc-nd/4.0/.

\section{References}

1. Haugen BR, Alexander EK, Bible KC, et al. 2015 American Thyroid Association Management Guidelines for adult patients with thyroid nodules and differentiated thyroid cancer: the American Thyroid Association Guidelines Task Force on Thyroid Nodules and Differentiated Thyroid Cancer. Thyroid 2016;26:1-133.

2. Nixon IJ, Whitcher MM, Palmer FL, et al. The impact of distant metastases at presentation on prognosis in patients with differentiated carcinoma of the thyroid gland. Thyroid 2012;22:884-9.

3. Sabet A, Binse I, Dogan S, et al. Distinguishing synchronous from metachronous manifestation of distant metastases: a prognostic feature in differentiated thyroid carcinoma. Eur J Nucl Med Mol Imaging 2017;44:190-5.

4. Kim H, Park SY, Jung J, et al. Improved survival after early detection of asymptomatic distant metastasis in patients with thyroid cancer. Sci Rep 2019;9:18745. 
5. Filetti S, Durante C, Hartl D, et al. Thyroid cancer: ESMO Clinical Practice Guidelines for diagnosis, treatment and follow-up†. Ann Oncol 2019;30:1856-83.

6. Janovitz T, Barletta JA. Clinically relevant prognostic parameters in differentiated thyroid carcinoma. Endocr Pathol 2018;29:357-64.

7. Bae JS, Kim Y, Jeon S, et al. Clinical utility of TERT promoter mutations and ALK rearrangement in thyroid cancer patients with a high prevalence of the BRAF V600E mutation. Diagn Pathol 2016;11:21.

8. Landa I, Ganly I, Chan TA, et al. Frequent somatic TERT promoter mutations in thyroid cancer: higher prevalence in advanced forms of the disease. J Clin Endocrinol Metab 2013;98:E1562-6.

9. Melo M, da Rocha AG, Vinagre J, et al. TERT promoter mutations are a major indicator of poor outcome in differentiated thyroid carcinomas. J Clin Endocrinol Metab 2014;99:E754-65.

10. Sohn SY, Park WY, Shin HT, et al. Highly concordant key genetic alterations in primary tumors and matched distant metastases in differentiated thyroid cancer. Thyroid 2016;26:672-82.

11. Mihailovic JM, Stefanovic LJ, Malesevic MD, et al. Metastatic differentiated thyroid carcinoma: clinical management and outcome of disease in patients with initial and late distant metastases. Nucl Med Commun 2009;30:558-64.

12. Shoup M, Stojadinovic A, Nissan A, et al. Prognostic indicators of outcomes in patients with distant metastases from differentiated thyroid carcinoma. J Am Coll Surg 2003;197:191-7.

13. Parameswaran R, Shulin Hu J, Min En N, et al. Patterns of metastasis in follicular thyroid carcinoma and the difference between early and delayed presentation. Ann R Coll Surg Engl 2017;99:151-4.

14. Jung CK, Jung SH, Jeon S, et al. Risk stratification using a novel genetic classifier including PLEKHS1 promoter mutations for differentiated thyroid cancer with distant metastasis. Thyroid 2020. [Epub ahead of print].

15. Wassermann J, Bernier MO, Spano JP, et al. Outcomes and prognostic factors in radioiodine refractory differentiated thyroid carcinomas. Oncologist 2016;21:50-8.

16. Miyauchi A, Kudo T, Miya A, et al. Prognostic impact of serum thyroglobulin doubling-time under thyrotropin suppression in patients with papillary thyroid carcinoma who underwent total thyroidectomy. Thyroid 2011;21:707-16.

17. Cho U, Mete O, Kim MH, et al. Molecular correlates and rate of lymph node metastasis of non-invasive follicular thyroid neoplasm with papillary-like nuclear features and invasive follicular variant papillary thyroid carcinoma: the impact of rigid criteria to distinguish non-invasive follicular thyroid neoplasm with papillary-like nuclear features. Mod Pathol 2017;30:810-25.

18. Jung CK, Kim Y, Jeon S, et al. Clinical utility of EZH1 mutations in the diagnosis of follicular-patterned thyroid tumors. Hum Pathol 2018;81:9-17.

19. Seo JY, Park JH, Pyo JY, et al. A Multi-institutional study of prevalence and clinicopathologic features of noninvasive follicular thyroid neoplasm with papillary-like nuclear features (NIFTP) in Korea. J Pathol Transl Med 2019;53:378-85.

20. Kim SY, Kim T, Kim K, et al. Highly prevalent BRAF V600E and low-frequency TERT promoter mutations underlie papillary thyroid carcinoma in Koreans. J Pathol Transl Med 2020;54:310-7.

21. Albano D, Panarotto MB, Durmo R, et al. Clinical and prognostic role of detection timing of distant metastases in patients with differentiated thyroid cancer. Endocrine 2019;63:79-86.

22. Papp S, Asa SL. When thyroid carcinoma goes bad: a morphological and molecular analysis. Head Neck Pathol 2015;9:16-23.

23. Landa I, Ibrahimpasic T, Boucai L, et al. Genomic and transcriptomic hallmarks of poorly differentiated and anaplastic thyroid cancers. J Clin Invest 2016;126:1052-66.

24. Xu B, Ghossein R. Genomic landscape of poorly differentiated and anaplastic thyroid carcinoma. Endocr Pathol 2016;27:205-12.

25. Amin MB, Edge S, Greene F, et al. AJCC cancer staging manual. 8th ed. New York: Springer, 2017:873-90.

26. Cancer Genome Atlas Research Network. Integrated genomic characterization of papillary thyroid carcinoma. Cell 2014;159:676-90.

27. Yang J, Gong Y, Yan S, et al. Association between TERT promoter mutations and clinical behaviors in differentiated thyroid carcinoma: a systematic review and meta-analysis. Endocrine 2020;67:44-57.

28. Lloyd RV, Osamura RY, Klöppel G, et al. WHO classification of tumors of endocrine organs. 4th ed. Lyon: International Agency for Research on Cancer (IARC), 2017:65-91.

29. Liu R, Bishop J, Zhu G, et al. Mortality risk stratification by combining BRAF V600E and TERT promoter mutations in papillary thyroid cancer: genetic duet of BRAF and TERT promoter mutations in thyroid cancer 
mortality. JAMA Oncol 2017;3:202-8.

30. Liu X, Qu S, Liu R, et al. TERT promoter mutations and their association with BRAF V600E mutation and aggressive clinicopathological characteristics of thyroid cancer. J Clin Endocrinol Metab 2014;99:E1130-6.

31. Liu X, Bishop J, Shan Y, et al. Highly prevalent TERT promoter mutations in aggressive thyroid cancers. Endocr Relat Cancer 2013;20:603-10.

32. Howlader N, Noone AM, Krapcho M, et al. SEER Cancer Statistics Review, 1975-2017, National Cancer Institute. Bethesda, MD, https://seer.cancer.gov/csr/1975_2017/, based on November 2019 SEER data submission, posted to the SEER web site, April 2020.

33. Kim H, Kim HI, Kim SW, et al. Prognosis of differentiated thyroid carcinoma with initial distant metastasis: a multicenter study in Korea. Endocrinol Metab (Seoul) 2018;33:287-95

34. Rössing RM, Jentzen W, Nagarajah J, et al. Serum thyroglobulin doubling time in progressive thyroid cancer. Thyroid 2016;26:1712-8.

Cite this article as: Jung CK, Lee S, Bae JS, Lim DJ. Lateonset distant metastases confer poor prognosis in patients with well-differentiated thyroid cancer. Gland Surg 2020;9(5):18571866. doi: 10.21037/gs-20-416
35. Iwasaki H, Yamazaki H, Takasaki H, et al. Treatment outcomes of differentiated thyroid cancer with distant metastasis improve by tyrosine kinase inhibitors. Oncol Lett 2019;17:5292-300.

36. Yin N, Sherman SI, Pak Y, et al. The de novo detection of anti-thyroglobulin antibodies and differentiated thyroid cancer recurrence. Thyroid 2020;30:1490-5.

37. Spencer C, Fatemi S. Thyroglobulin antibody (TgAb) methods - Strengths, pitfalls and clinical utility for monitoring TgAb-positive patients with differentiated thyroid cancer. Best Pract Res Clin Endocrinol Metab 2013;27:701-12.

38. Sampson E, Brierley JD, Le LW, et al. Clinical management and outcome of papillary and follicular (differentiated) thyroid cancer presenting with distant metastasis at diagnosis. Cancer 2007;110:1451-6.

39. Schlumberger M, Leboulleux $S$. Treatment of distant metastases from follicular cell-derived thyroid cancer. F1000Prime Rep 2015;7:22. 